\title{
Bovine tuberculosis in Northern Ireland: quantification of the population disease-level effect from cattle leaving herds detected as a source of infection
}

\author{
L. P. DOYLE ${ }^{1 *}$, E. A. COURCIER ${ }^{1}$, A. W. GORDON ${ }^{2}$, M. J. H. O'HAGAN ${ }^{1}$, \\ J. A. STEGEMAN ${ }^{3}$ AND F. D. MENZIES ${ }^{1}$ \\ ${ }^{1}$ Veterinary Epidemiology Unit, Department of Agriculture, Environment and Rural Affairs, Dundonald House, \\ Upper Newtownards Road, Belfast BT4 3SB, UK \\ ${ }^{2}$ Biometrics Branch, Agri-Food and Biosciences Institute, Newforge Lane, Belfast, BT9 5PX, UK \\ ${ }^{3}$ Department of Farm Animal Health, Faculty of Veterinary Medicine, University of Utrecht, Yalelaan 7 , \\ Utrecht, The Netherlands
}

Received 13 March 2017; Final revision 25 August 2017; Accepted 12 October 2017; first published online 6 November 2017

\section{SUMMARY}

Determination of the proportion of bovine tuberculosis (bTB) breakdowns attributed to a herd purchasing infected animals has not been previously quantified using data from the Animal and Public Health Information System (APHIS) database in Northern Ireland. We used a casecontrol study design to account for the infection process occurring in the disclosing bTB breakdown herds. Cases $(N=6926)$ were cattle moving to a future confirmed bTB breakdown where they would disclose as a confirmed bTB reactor or a Lesion at Routine Slaughter (LRS). Controls $(N=303499)$ were cattle moving to a future confirmed bTB breakdown where they did not become a bTB reactor or LRS. Our study showed that the cattle leaving herds which disclosed bTB within 450 days had an increased odds of becoming a confirmed bTB reactor or LRS compared with the cattle which left herds that remained free for 450 days (odds ratio (OR) $=2 \cdot 09: 95 \%$ CI 1.96-2.22). Of the 12060 confirmed bTB breakdowns included in our study (2007-2015 inclusive), 31\% (95\% CI 29.8-31.5) contained a confirmed bTB reactor(s) or LRS(s) at the disclosing test which entered the herd within the previous 450 days. After controlling for the infection process occurring in the disclosing bTB breakdown herd, our study showed that $6 \cdot 4 \%(95 \%$ CI $5 \cdot 9-6 \cdot 8)$ of bTB breakdowns in Northern Ireland were directly attributable to the movement of infected animals.

Key words: Case-control study, epidemiology, movement, cattle, Mycobacterium bovis.

\section{INTRODUCTION}

Tuberculosis is an infectious disease that affects practically all vertebrates and is caused by acid-fast bacilli of the genus Mycobacterium. Classically it is defined

\footnotetext{
* Author for correspondence: L. P. Doyle, Veterinary Epidemiology Unit, Department of Agriculture, Environment and Rural Affairs, Dundonald House, Upper Newtownards Road, Belfast BT4 3SB, UK.

(Email: liam.doyle@daera-ni.gov.uk)
}

as a chronic debilitating disease; however, it occasionally assumes an acute and rapidly progressive course. Three main types of tubercle bacilli are recognised: human, bovine and avian with Mycobacterium bovis being the common cause of tuberculosis in cattle [1].

In Northern Ireland, first attempts at control of bovine tuberculosis (bTB) began in 1935 (compulsory since 1959) and despite the current programme of regular testing and abattoir surveillance, tuberculosis has remained endemic in the Northern Ireland cattle 
population ( 1.6 million animals). Within this population, there are approximately 285000 dairy cows and 279000 beef cows residing in approximately 23 000 herds with average size of 64 animals [2]. During 2015, the annual herd incidence was $7 \cdot 15 \%$ (equating to 2095 herds with bTB reactors) with an annual animal incidence of $0.66 \%$ (equating to 11 002 bTB reactor animals). Abernethy et al. [3] provided a comprehensive review of the Northern Ireland bTB eradication programme and more detailed statistics on bTB can be found on the DAERA (Department of Agriculture, Environment and Rural Affairs) web site [4].

Movement of infected cattle between herds is considered an important route of dissemination of bTB. The proportion of bTB herd breakdowns attributed to the purchase of infected cattle has been estimated in Ireland (6-7\%) and Great Britain $(16 \%)[5,6]$. In Northern Ireland, although purchase of beef/store animals from herds that had a bTB breakdown in the last 3 years was shown to be a risk factor for a bTB breakdown, no quantification of the effect at a national level was calculated [7]. Other studies that have investigated cattle movement as a risk factor for bTB breakdowns have looked at different aspects of this complicated issue [5, 6, 8-12]. Risk factors that have been demonstrated include cattle movements from markets and farm sales [8, 12], cattle purchased from herds with a higher risk of having bTB [10] and the severity of the original bTB breakdown [11].

Purchased cattle can either be infected with bTB prior to joining the recipient herd or become infected during their residency [13]. Previous studies have not taken the latter post-movement exposure into account within their study design [5, 6, 8-13]. The case-control study presented in this paper was designed to overcome this issue by ensuring that the cases and controls were exposed to the same temporal and environmental conditions and hence negating against effects while resident in the recipient herd.

This study used the Northern Ireland bTB surveillance programme to investigate the contribution of cattle movement to overall bTB infection levels.

\section{Study objective}

The objective of this case-control study was to quantify the likelihood that the movement of bTB-infected animals were responsible for future bTB-confirmed herd breakdowns and to determine their impact at a population level.

\section{MATERIALS AND METHODS}

\section{Study population and study design}

The study population consisted of cattle moving to recipient herds, which would become confirmed bTB breakdowns, with explanatory variables based on their previous donor herds. A confirmed bTB breakdown was defined as a herd with at least one confirmed bTB reactor or a herd with an animal at routine slaughter that was confirmed as having bTB through histological or bacteriological examination (Lesion at Routine Slaughter, LRS). A confirmed bTB reactor was defined as an animal that had been interpreted as having a positive result to the single intradermal comparative tuberculin test (SICTT) and was also found to have bTB-type lesions at postmortem examination and/or was diagnosed as bTB through histological or bacteriological examination. The disclosure date of a confirmed bTB breakdown was the date of a SICTT at which a confirmed bTB reactor was found or the SICTT herd test date after disclosure of the LRS.

The cattle traced to confirmed bTB breakdowns formed the cases and controls for the study. Cases $(N=6926)$ were traced cattle that had entered a herd that would become a confirmed bTB breakdown where they would disclose as a confirmed bTB reactor or LRS. Controls $(N=303499)$ were cattle that had entered a herd that would become a confirmed bTB breakdown but did not disclose as a confirmed bTB reactor or LRS.

The cattle movements eligible for inclusion in the study occurred in the period 1 January 2007 to 1 August 2014. This time period allowed for a minimum of 450 days follow-up of the donor herds after the departure of the case/control animals (e.g. from 1 August 2014 to 1 January 2016).

In this study, the risk window for each bTB herd breakdown was initiated on the date of receipt of the first case animal and finished on the date the herd became a confirmed bTB breakdown. This risk window was used to identify cases and controls that joined the recipient herd. The risk window was limited to a maximum duration of 450 days ( 15 months). The value of 450 days was used as it is an absolute limit placed on inter-herd test intervals within the Northern Ireland bTB eradication programme.

For each of the cases and controls, all herds in which they had been resident during the 450 days prior to their associated risk window disclosure date were identified. Any herd supplying both case and 
control animals was excluded from the analysis. Explanatory variables for this study were based on the herds in which study animals were resident in prior to moving to their respective risk windows.

The design compared the number of bTB breakdowns in herds through which case animals had resided to the number of bTB breakdowns in herds through which control animals had resided (Fig. 1). Thus, whether the herds supplying animals into risk windows have a bTB breakdown within a certain time period provides information on the previous environment of the case or control animals. Cases were resident in one or more herds, before arrival in the bTB breakdown herd and either entered into the risk window already infected or became infected in the bTB breakdown herd. If a higher proportion of animals became infected within the trace herds compared with the controls, then the rate of bTB breakdown in herds supplying cases will be greater than those supplying controls. Such a finding would indicate the possible need to enhance the bTB eradication programme measures in mitigation against this risk to recipient herds.

\section{Data collection and variable definitions}

The identification, movement and test history of all individual animals were extracted from the Animal and Public Health Information System (APHIS), which is managed by DAERA [14].

Explanatory variables defined for the dataset and linked to the herds supplying study animals where disclosure of a bTB breakdown $<450$ days after the study animal had left the herd and number of days to the closest bTB herd test after the animal movement. Other explanatory variables include local area bTB incidence in the year of the animal movement, herd size, movement intensity into the herd in the 90 days prior to the animal movement, herd type, bTB herd history and divisional veterinary office (DVO; $n=10$ ). Disclosure of a bTB breakdown $<450$ days after the animal had left a herd was included as a binary variable. Number of days to the closest bTB herd test after the animal movement was allocated a unit of per 30 days, thus representing a monthly effect. This variable was included to account for the fact that within the Northern Ireland bTB testing programme herds moving case animals will be more likely to have a tracing test imposed compared with the herds moving control animals. Local area bTB incidence in the year of animal movement from a herd was calculated based on a geographical area called a patch, which is a subdivision of a DVO that comes under the administration of a Veterinary Officer (VO). The local area bTB incidence was calculated as the number of new bTB breakdowns (breakdowns could be initiated by bTB reactor disclosure at or confirmed LRS) to total herds bTB tested in the patch in each of the study years and expressed as a percentage. Thus for each patch, the bTB incidence in the year of study animal movement from a herd was used in the analysis. Herd size was determined as the number of cattle tested in the trace herd at the first bTB herd test after movement of the study animal. Movement intensity was defined as the number of cattle purchased into a herd in the 90 days before the study animal left the trace herd and was measured in units of animals per year. Movement intensity was categorised to five levels so as to create a zero category for closed herds and four other levels containing approximately equal numbers of observations. The zero-level category selected situations into which no cattle movement occurred in the 90 days prior to the study animal leaving a herd and represented closed herd management systems. Herd type was either dairy or non-dairy with the former defined as having a milk licence and this was included in the study as a binary variable. BTB herd history was defined as the presence of a bTB breakdown (presence of bTB reactor(s) or LRS (s)) during the 2-year period prior to the movement of a study animal from the herd and was included in the study as a binary variable. DVO was a naturally categorised variable representing the 10 local veterinary administrative areas.

\section{Data analysis}

Microsoft Access ${ }^{\mathrm{TM}}$ (Microsoft Corporation, Redmond, WA, USA) and R Version 2.15.0 (The R Foundation for Statistical Computing) were used for all data manipulations and StataIC $12^{1}$ was used for data analysis. Summary statistics were calculated for each variable and an initial descriptive analysis of the dataset completed to provide background information. The model framework used in this study was binary logistic regression using clustered robust standard errors. The response variable was animals disclosing with bTB (cases) or not disclosing (controls) after moving from donor herds to the risk window of

\footnotetext{
${ }^{1}$ StataCorp. 2011. Stata Statistical Software: Release 12. College
} Station, TX: StataCorp LP. 


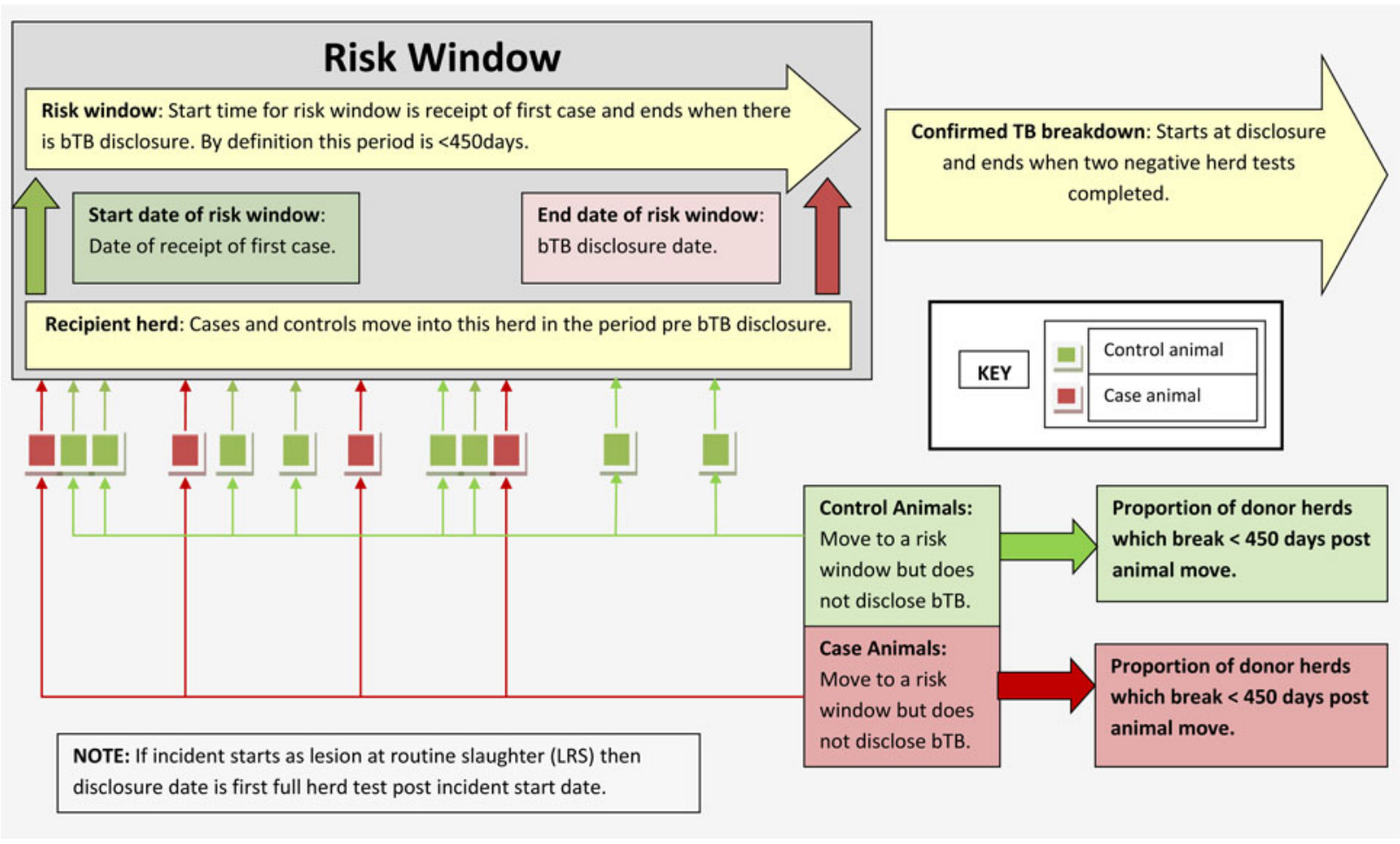

Fig. 1. Pictorial representation of study design.

their recipient herd. Explanatory variables for the analysis were as previously described. The cluster term used in the analysis was the identifier (official tag number) for each study animal. Use of this identifier grouped the herds through which a study animal moved on their way to a recipient herd. For example, if an animal moved through two herds prior to it entering the recipient herd, its identifier linked these two herds into the cluster term.

The variables number of days to the closest bTB herd test, local area bTB incidence and herd size were assessed for their inclusion in the analysis as continuous, without categorisation. This was initially carried out visually by inspecting their lowess (locally weighted scatter plot smoothing) curves with a superimposed linear predictor. Any of the linear predictors which showed departures from the lowess curves were modelled in more detail by spline regression to determine if piecewise representations provided better outcome predictors. If a piecewise representation could be shown to provide a better model than the full linear predictor for any of the continuous variables, then the variable was categorised at the cut-off points [15].

An initial univariable analysis was applied to each variable to determine if they were statistically significant, at the $P=0 \cdot 2$ level. As all variables initially chosen for the study were considered important potential risk factors, they were not immediately removed on the basis of the univariable analysis result. Instead a full multivariable model with all study variables was fitted, so that any variable non-significant in the univariable analysis at the $P=0.2$ level and nonsignificant at the $P=0.05$ level in the multivariable model would be removed. The variables selected by this process formed the new full model. In the new full model, each of the explanatory variables was in turn individually removed to produce a series of reduced models; each containing one explanatory variable less than the full model. Each reduced model was compared for fit to the full model using Bayesian information criterion (BIC) comparisons. If a reduced model provided a better fit than the full model (i.e. if the reduced model had a lower BIC value), then the specified explanatory variable was removed from the analysis [16].

All biologically plausible two-way interactions were assessed to determine if their addition improved overall model fit. Any interaction which improved the model fit by lowering the BIC value was selected for inclusion in the final model [16]. A final multivariable model including selected interactions was compared with the original full model, without interactions to determine if their inclusion improved overall fit. A correlation matrix of all pair wise combinations of 
variables assessed using Pearson product-moment correlation coefficients implemented in STATA ${ }^{\mathrm{TM}}$ was carried out to assess collinearity. The finalised model was subjected to a Hosmer-Lemeshow goodness-of-fit test (decile sub-grouped) to determine how well it fitted the data.

We calculated the attributable fraction $\left(\mathrm{AF}_{\mathrm{e}}\right)$ (adjusted for interaction terms) of bTB infection in individuals exposed to the risk factor of leaving herds which disclosed bTB reactors or LRSs within 450 days [17]. The population attributable fraction (PAF) was also calculated for diseased animals (cattle disclosing as bTB reactors or LRSs in confirmed bTB breakdowns) attributable to the risk factor of them leaving herds which had a breakdown within 450 days which in turn allowed calculation of the number of cases attributed to it [17]. As these cases were either cattle disclosing as bTB reactors or LRSs in confirmed bTB breakdowns, if each animal went individually to a different recipient herd, they would have the potential to generate the same number of breakdowns. However, any group of purchased cattle will disperse to recipient herds as singles, doubles, triples, etc. meaning they will move to fewer herds than the total number of animals. To account for this effect, we applied the movement distribution for all cases to the sub-group selected as the PAF to determine the number of bTB breakdowns during the study period which received diseased animals.

\section{RESULTS}

In this case-control study, there were 6926 cases and 303499 controls drawn from all the Northern Ireland bTB testing and movement data in the study 2007-2015 inclusive. During this period, there were 12060 bTB confirmed breakdowns, of which 3695 $(31 \%)$ had risk windows with case animals. Removal of risk windows that included herds which supplied both case and control animals left 2958 (24\%) eligible risk windows. Of the remaining breakdowns, 6773 $(56 \%)$ had only control animals purchased during the 450-day period prior to breakdown initiation, while $1592(13 \%)$ had not purchased any animals during the risk window.

Of the three continuous variables assessed for categorisation (number of days to the closest bTB herd test, local area bTB incidence and herd size), only herd size was categorised. Herd size was categorised at the levels $0-50,51-100,101-150,151-300$ and $>300$ as piecewise representation using spline regression at these cut-off points provided a better fit of the data than the continuous representation of the variable.

In the univariable analysis, statistically significant variables $(P<0 \cdot 2)$ were bTB herd disclosure within 450 days after the animal left the herd, number of days to closest bTB herd test, local area bTB incidence, herd size, movement intensity into the herd and local DVO of the case or control animal prior to move out. The variables herd type and bTB herd histories were not statistically significant (Table 1).

In the multivariable analysis, all eight explanatory variables were statistically significant $(P<0.05)$; thus satisfying the selection criteria to be included in the full model. As a result of model fitting, the variables retained in the final model were bTB herd disclosure within 450 days after the animal left the herd, number of days to closest bTB herd test, local area bTB incidence, herd size, movement intensity, bTB herd history and DVO of the donor herd. Herd type was not included in the final model as its presence in the full model did not improve the fit over a reduced model. BTB herd history was not statistically significant in the univariable analysis but statistically significant in the multivariable analysis and its addition to the model was shown to increase overall model fit. As bTB herd history fulfilled the selection criteria, it was retained as part of the final model.

Included in the final multivariable model (Table 2) was a two-way interaction term acting between the variables bTB herd disclosure within 450 days after the animal left the herd and the time period to the closest bTB herd test after the animal move. This was the only interaction which improved model fit. Inclusion of the interaction term meant that the assessment of the odds ratio (OR) for the variable bTB herd disclosure within 450 days after animal movement required interpretation based on the time period to the closest bTB herd test (Fig. 2).

For a time period to the closest bTB test of 111 days (median of the variable; IQR 56-189), cattle leaving herds which disclosed bTB within 450 days compared with the cattle that left herds that did not disclose bTB were associated with an odds of becoming a bTB reactor or LRS of 2.09 (95\% CI 1.96-2.22) and this odds decreased with increasing time since closest bTB test. For every $1 \%$ increase in local area bTB incidence, the odds of an animal becoming a bTB reactor or LRS in the recipient herd also increased $(\mathrm{OR}=$ 1.02: $95 \%$ CI 1.01-1.03). Regarding herd size, the odds of an animal becoming a bTB reactor or LRS 
Table 1. Results from the univariable animal movement case-control study with each of the explanatory variables used as a single fixed level term

\begin{tabular}{|c|c|c|c|c|c|c|c|c|}
\hline \multirow[b]{2}{*}{ Variable } & \multirow[b]{2}{*}{ Exposure level } & \multirow[b]{2}{*}{ Odds ratio } & \multirow[b]{2}{*}{$95 \% \mathrm{CI}$} & \multicolumn{2}{|c|}{$\begin{array}{l}\text { Categorised variables: } \\
\text { number of case/controls } \\
\text { per level }\end{array}$} & \multicolumn{2}{|c|}{$\begin{array}{l}\text { Non-categorised variables: median } \\
\text { (range 5-95\%) }\end{array}$} & \multirow[b]{2}{*}{$P$-value } \\
\hline & & & & Cases & Controls & Cases & Controls & \\
\hline \multirow{2}{*}{$\begin{array}{l}\text { bTB disclosure }<450 \text { days after animal } \\
\text { movement }\end{array}$} & No & $1 \cdot 00$ & - & 5231 & 264569 & - & - & \multirow[t]{2}{*}{$P<0 \cdot 001$} \\
\hline & Yes & $2 \cdot 20$ & $2 \cdot 08-2 \cdot 33$ & 1695 & 38930 & - & - & \\
\hline $\begin{array}{l}\text { Number of days to the closest bTB herd test } \\
\text { after animal movement }\end{array}$ & Per 30 days & $0 \cdot 92$ & $0 \cdot 91-0 \cdot 93$ & 6926 & 303499 & $111(14-320)$ & $142(16-347)$ & $P<0.001$ \\
\hline $\begin{array}{l}\text { Local area incidence: year of animal } \\
\text { movement }(\%)\end{array}$ & Per extra $1 \%$ & $1 \cdot 05$ & $1 \cdot 05-1 \cdot 06$ & 6926 & 303499 & $7 \cdot 13(3 \cdot 71-16 \cdot 17)$ & $6 \cdot 71(3 \cdot 21-14 \cdot 74)$ & $P<0.001$ \\
\hline \multirow[t]{5}{*}{ Herd size } & $0-50$ & $1 \cdot 00$ & - & 2949 & 124840 & - & - & \multirow[t]{5}{*}{$P=0 \cdot 001$} \\
\hline & $51-100$ & $0 \cdot 93$ & $0 \cdot 87-0.99$ & 1582 & 72050 & - & - & \\
\hline & $101-150$ & $0 \cdot 91$ & $0 \cdot 84-0 \cdot 98$ & 822 & 38306 & - & - & \\
\hline & $151-300$ & $1 \cdot 01$ & $0.94-1 \cdot 08$ & 1145 & 48013 & - & - & \\
\hline & $>300$ & $0 \cdot 89$ & $0 \cdot 81-0 \cdot 99$ & 428 & 20290 & - & - & \\
\hline \multirow{5}{*}{$\begin{array}{l}\text { Herd purchase intensity in the } 3 \text { months } \\
\text { prior to study animal movement (animals/ } \\
\text { year) }\end{array}$} & 0 & $1 \cdot 00$ & - & 2548 & 124303 & - & - & \multirow[t]{5}{*}{$P<0.001$} \\
\hline & $1-5$ & $1 \cdot 17$ & $1 \cdot 10-1 \cdot 25$ & 1764 & 73325 & - & - & \\
\hline & $6-30$ & $1 \cdot 11$ & $1 \cdot 04-1 \cdot 19$ & 1339 & 58806 & - & - & \\
\hline & $31-100$ & $1 \cdot 10$ & $1 \cdot 01-1 \cdot 21$ & 557 & 24594 & - & - & \\
\hline & $>100$ & $1 \cdot 56$ & $1.44-1.69$ & 718 & 22471 & - & - & \\
\hline \multirow[t]{2}{*}{ Herd type } & Non-dairy & $1 \cdot 00$ & - & 5240 & 231052 & - & - & \multirow[t]{2}{*}{$P=0 \cdot 368$} \\
\hline & Dairy & $1 \cdot 03$ & $0.97-1.09$ & 1686 & 72447 & - & - & \\
\hline \multirow{2}{*}{$\begin{array}{l}\text { Presence of a bTB breakdown in the } 2 \text { years } \\
\text { prior to study animal movement }\end{array}$} & No & $1 \cdot 00$ & - & 5847 & 256056 & - & - & \multirow[t]{2}{*}{$P=0.910$} \\
\hline & Yes & $1 \cdot 00$ & $0.93-1 \cdot 06$ & 1097 & 47434 & - & - & \\
\hline \multirow[t]{10}{*}{ DVO of herd of study animal } & 1 & $1 \cdot 00$ & - & 548 & 24519 & - & - & \multirow[t]{10}{*}{$P<0 \cdot 001$} \\
\hline & 2 & $0 \cdot 64$ & $0 \cdot 55-0 \cdot 74$ & 318 & 22342 & - & - & \\
\hline & 3 & $0 \cdot 93$ & $0 \cdot 82-1 \cdot 05$ & 704 & 34013 & - & - & \\
\hline & 4 & $0 \cdot 83$ & $0 \cdot 74-0 \cdot 94$ & 671 & 35977 & - & - & \\
\hline & 5 & $1 \cdot 35$ & $1 \cdot 21-1 \cdot 51$ & 1312 & 43395 & - & - & \\
\hline & 6 & $0 \cdot 82$ & $0 \cdot 72-0 \cdot 93$ & 471 & 25757 & - & - & \\
\hline & 7 & 0.63 & $0 \cdot 52-0 \cdot 77$ & 158 & 11133 & - & - & \\
\hline & 8 & $1 \cdot 40$ & $1 \cdot 25-1 \cdot 56$ & 1100 & 35152 & - & - & \\
\hline & 9 & $1 \cdot 49$ & $1 \cdot 32-1 \cdot 68$ & 716 & 21546 & - & - & \\
\hline & 10 & $0 \cdot 84$ & $0 \cdot 75-0 \cdot 94$ & 928 & 49665 & - & - & \\
\hline
\end{tabular}

DVO, divisional veterinary office. 
Table 2. Results from the final multivariable animal movement case-control study

\begin{tabular}{|c|c|c|c|}
\hline Variable & $\begin{array}{l}\text { Exposure } \\
\text { level }\end{array}$ & $\begin{array}{l}\text { Odds } \\
\text { ratio } \\
\end{array}$ & $\begin{array}{l}\text { 95\% Confidence } \\
\text { - interval }\end{array}$ \\
\hline \multirow[t]{2}{*}{ bTB disclosure $<450$ days after animal movement } & No & $1 \cdot 00$ & - \\
\hline & Yes & $2 \cdot 85$ & $2 \cdot 60-3 \cdot 12$ \\
\hline Number of days to the closest bTB herd test after animal movement & Per 30 days & $0 \cdot 95$ & $0 \cdot 94-0 \cdot 96$ \\
\hline $\begin{array}{l}\text { [bTB disclosure }<450 \text { days }] \times[\text { number of days to the closest bTB herd test after } \\
\text { study animal movement] }\end{array}$ & Per 30 days & $0 \cdot 92$ & $0 \cdot 90-0.94$ \\
\hline Local area incidence: year of study animal movement $(\%)$ & Per extra $1 \%$ & $1 \cdot 02$ & $1 \cdot 01-1 \cdot 03$ \\
\hline \multirow[t]{5}{*}{ Herd size } & $0-50$ & $1 \cdot 00$ & - \\
\hline & $51-100$ & $0 \cdot 86$ & $0 \cdot 81-0 \cdot 92$ \\
\hline & $101-150$ & 0.79 & $0 \cdot 73-0 \cdot 85$ \\
\hline & $151-300$ & $0 \cdot 84$ & $0 \cdot 78-0 \cdot 90$ \\
\hline & $>300$ & $0 \cdot 70$ & $0 \cdot 63-0 \cdot 78$ \\
\hline \multirow{5}{*}{$\begin{array}{l}\text { Herd purchase intensity in the } 3 \text { months prior to study animal movement } \\
\text { (animals/year) }\end{array}$} & 0 & $1 \cdot 00$ & \\
\hline & 1 to 5 & $1 \cdot 19$ & $1 \cdot 12-1 \cdot 27$ \\
\hline & 6 to 30 & $1 \cdot 13$ & $1 \cdot 05-1 \cdot 21$ \\
\hline & 31 to 100 & $1 \cdot 11$ & $1 \cdot 01-1 \cdot 22$ \\
\hline & $>100$ & $1 \cdot 46$ & $1 \cdot 37-1 \cdot 59$ \\
\hline \multirow[t]{2}{*}{ Presence of a bTB breakdown in the 2 years prior to study animal movement } & No & $1 \cdot 00$ & - \\
\hline & Yes & $0 \cdot 85$ & $0 \cdot 80-0 \cdot 92$ \\
\hline \multirow[t]{10}{*}{ DVO of herd of study animal } & 1 & $1 \cdot 00$ & - \\
\hline & 2 & $0 \cdot 68$ & $0 \cdot 58-0 \cdot 78$ \\
\hline & 3 & 0.95 & $0 \cdot 84-1 \cdot 08$ \\
\hline & 4 & $0 \cdot 87$ & $0.77-0.98$ \\
\hline & 5 & $1 \cdot 35$ & $1 \cdot 21-1 \cdot 51$ \\
\hline & 6 & $0 \cdot 93$ & $0 \cdot 81-1 \cdot 06$ \\
\hline & 7 & $0 \cdot 72$ & $0 \cdot 59-0 \cdot 87$ \\
\hline & 8 & $1 \cdot 27$ & $1 \cdot 14-1 \cdot 43$ \\
\hline & 9 & $1 \cdot 35$ & $1 \cdot 18-1 \cdot 53$ \\
\hline & 10 & $0 \cdot 85$ & $0.76-0.95$ \\
\hline
\end{tabular}

DVO, divisional veterinary office.

was lower in all the larger herd size categories compared with the base-level category of $0-50$ cattle (Table 2). Increased purchasing (movement) intensity showed increased odds of an animal leaving the herd becoming a bTB reactor or LRS. At a purchase intensity of $>100$ animals per year in the 3 months prior to an animal leaving the herd, the odds of it becoming a bTB reactor or LRS increased compared with animals leaving herds which did not purchase animals $(\mathrm{OR}=$ 1.46: 95\% CI 1.37-1.59). With herds that had a bTB breakdown in the 2 years prior to an animal movement, there was a decreased odds of becoming a bTB reactor or LRS compared with the cattle leaving herds with no bTB (OR $=0 \cdot 85: 95 \%$ CI $0 \cdot 80-0 \cdot 92)$. Animals moving from herds in DVO Enniskillen, DVO Newry and DVO Newtownards (DVO5, DVO8 and DVO9) relative to DVO Armagh (DVO1) had the greatest increase in odds of becoming a bTB reactor or LRS (OR $=1 \cdot 35: 95 \%$ CI $1 \cdot 21-1 \cdot 51$, $\mathrm{OR}=1 \cdot 27: 95 \% \mathrm{CI} 1 \cdot 14-1 \cdot 43$ and $\mathrm{OR}=1 \cdot 35: 95 \% \mathrm{CI}$ $1 \cdot 18-1 \cdot 53$, respectively).

Collinearity assessment between variables showed there to be no linear correlations between variables with a correlation coefficient in excess of $0 \cdot 47$, which was recorded between the variables herd type and herd size. The Hosmer-Lemeshow goodness-of-fit test indicated a reasonable fit for the full multivariable model $(P=0 \cdot 42)$.

The $\mathrm{AF}_{\mathrm{e}}$ in individuals exposed to the risk factor of leaving herds which disclosed bTB reactors or LRSs within 450 days was determined using the OR returned in the multivariable model adjusted for the interaction term $\left(\mathrm{OR}=2 \cdot 09: 95 \% \mathrm{CI} 1 \cdot 96-2 \cdot 22 ; \mathrm{AF}_{\mathrm{e}}\right.$ $=0.52: 95 \%$ CI $0 \cdot 49-0 \cdot 55$ ). Thus, $52 \%$ of the bTB infection in individuals leaving herds which disclosed bTB reactors or LRSs within 450 days was accounted 
Interaction relationship between variables BTB disclosure $<450$ days and Number of days to closest herd test post case/control animal move

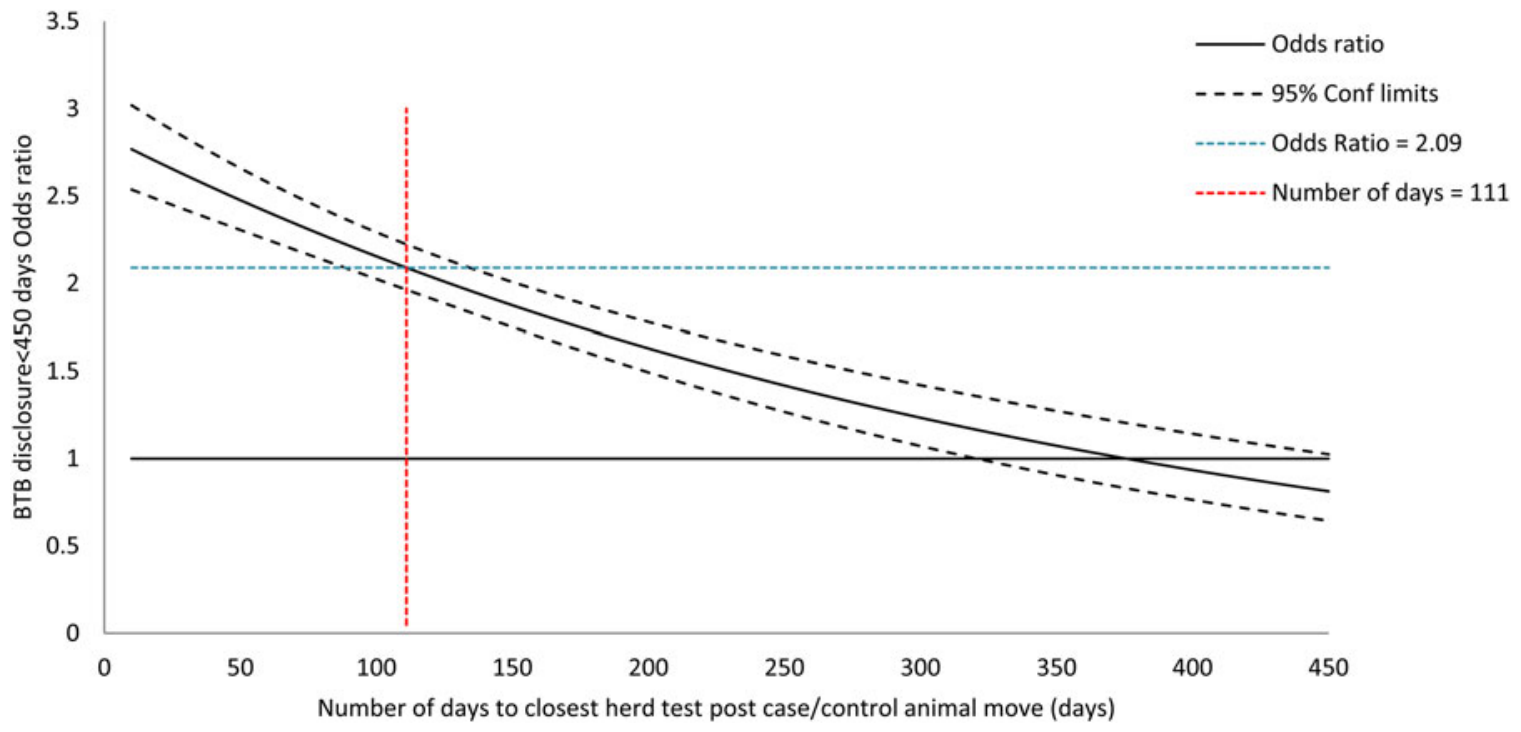

Fig. 2. The odds ratios for study variable bTB disclosure $<450$ days in the presence of an interaction with the number of days to the closest bTB herd test after animal movement.

for by this exposure. Given that there were 6926 cases and 1695 of them had left herds which had a bTB breakdown within 450 days, the PAF was calculated as $0 \cdot 13(95 \%$ CI $0 \cdot 12-0 \cdot 14)$, or in terms of diseased cattle which leave donor herds and enter the risk window of recipients where they will disclose as bTB skin reactors or LRSs, this has a value $(6926 \times 0 \cdot 13) 900$ animals. If the 900 cattle had moved out to the same distribution of bTB breakdowns as all other case animals, then 767 breakdowns could be attributed to this source, or $6.4 \%$ (767 of 12060 confirmed bTB breakdowns; 95\% CI 5.9-6.8) of the confirmed bTB breakdowns during the 9-year period of the study. Of the 12 060 confirmed bTB breakdowns, 3695 had an associated risk window, meaning that $31 \%(95 \%$ CI 29.8-31.5) of the bTB breakdowns at disclosure contain an LRS(s) or bTB reactor(s) which had joined the herd within the previous 450 days. Given that $6.4 \%$ (95\% CI 5.9-6.8) of the confirmed bTB breakdowns receive infected animal(s) in the 450 days prior to the herd disclosure, the calculated percentage range of total bTB reactors/LRSs attributable to them was $1 \cdot 1 \%(95 \% \mathrm{CI} 1 \cdot 0-1 \cdot 1)$ to $28 \cdot 8 \%(95 \% \mathrm{CI}$ $28 \cdot 5-29 \cdot 1)$.

\section{DISCUSSION}

All confirmed bTB breakdowns in Northern Ireland currently receive an epidemiological investigation from their local $\mathrm{VO}$ as part of which a judgement is made as to the most likely infection source. Current figures indicate that approximately $14 \%$ of the confirmed bTB breakdowns had purchased bTB-infected animals as their infection source [18]. It must also be noted that the infection source could not be established in $31 \%$ of these confirmed bTB breakdowns [18]. Our result (6.4\% (95\% CI 5.9-6.8)) suggested that this might be an overestimation for purchased infection as a bTB source. The fact that $31 \%$ of disclosing bTB breakdowns purchased bTB reactor(s) or LRS(s) within the previous 450 days may have biased this as a choice of infection source.

Results of our study indicate that $6.4 \%$ (95\% CI 5.9 6.8) of the confirmed breakdowns can be directly attributed to the movement of infected animals. A similar value (determined using bTB herd restrictions) was found in Republic of Ireland (ROI) where 6-7\% of the current herd restrictions were attributed to the recent introduction of an infected animal [5]. Given the common environmental conditions and farm demographics between the ROI and Northern Ireland, agreement on this figure must provide some validation of the significance of this particular disease spread mechanism on the island of Ireland. The unique feature of our study design is the ability to control for the infection process that occurs after the animal leaves a donor/source herd. This design also allows quantification of infection levels in cattle as they move from donor to recipient herds. 
When a herd purchases an infected animal, it has the potential to spread infection onwards to other cattle within that herd. Respiratory excretion and inhalation of M. bovis is considered to be the main route by which cattle-to-cattle transmission occurs in bovines [19]. Other routes of infection for bTB include ingestion of M. bovis, which is considered a less common route, while udder infections which can lead to pseudo-vertical and congenital transmission are considered relatively rare $[19,20]$. Excretion of the infectious agent $M$. bovis is an intermittent process with periods ranging from 6 to 25 weeks [19]. Single time point sampling of nasal and tracheal mucus taken from reactors with grossly visible tuberculous lesions also indicated that at least $19 \%$ were shedding M. bovis [21]. Our study investigated movement of pre-infected animals; however, of equal interest was the potential consequence of onward cattle to cattle transmission. The ability of an infected animal to disseminate infection is not a continuous process with some animals being more effective disseminators than others [19]. This suggests variability at a herd level in potential outcomes from receipt of infected animals. However, given the nature of our data, we can say that the range of proportion of bTB reactors/LRSs attributable to purchased infected animals was between $1 \cdot 1 \%(95 \%$ CI $1 \cdot 0-1 \cdot 1)$ and $28 \cdot 8 \%(95 \%$ CI 28.5-29:1).

Movement of infected cattle is only one source of bTB for a herd. Within the UK, wildlife mainly in the form of badgers plays a widely debated role in the transmission and maintenance of bTB. The direct contribution of badgers to confirmed bTB cattle herd breakdowns in high incidence areas in England has been estimated to be $5 \cdot 7 \%$ (bootstrap $95 \%$ CI 0.9 $25 \%$ ) with an overall contribution of badgers, taking into account onward cattle-to-cattle transmission, of 54\% (overdispersion adjusted 95\% CI 38-66\%) [22]. This work suggests that even though the level of transmission from badgers in these high incidence areas $(5 \cdot 7 \%)$, which is similar to the level of transmission to herds from purchased animals in our study $(6 \cdot 4 \%)$, the onward cattle-to-cattle transmission from a badger source appears to be greater. This partly could be a reflection of management practices where purchased animals such as beef fattening cattle are batched in such a way that they have less access to other herd members reducing within-herd contact. Alternatively, farm biosecurity procedures may also limit within-herd spread from purchased animals.
Finding an effective method to prevent infected animals being traded from one herd to another would assist in disease control. It would prevent bTB breakdowns directly instigated by movement of infected animals and prevent onward cattle-to-cattle transmission in these receiving herds. One such method to reduce the movement of bTB-infected animals is a risk-based trading scheme where cattle herds are identified and ranked according to their risk of being or becoming infected [23, 24]. One such study, Adkin et al. identified herd history of bTB, local bTB incidence, herd size and movements of animals onto farms in batches from high-risk areas as being significantly associated with increased probability of bTB infection on a farm [24]. The risk factors were then used to award each farm trading cattle in England and Wales a risk score (rating 1-5) with the aim of providing information allowing avoidance of risky purchases. These data are also readily available for Northern Ireland and a similar methodology could help reduce the number of bTB-infected animals traded in Northern Ireland. Indeed, use of informed purchasing is a tool recommended within the recent bTB eradication strategy for Northern Ireland [25].

A limitation placed on this study is its dependence on information from the SICTT and abattoir surveillance. In Irish conditions, it was estimated that the sensitivity of the SICTT ranged between $68 \%$ and $95 \%$, while specificity ranged between $96 \%$ and $99 \%$, although more recent analyses would suggest that the sensitivity is lower than this $[5,26]$. With abattoir surveillance, it has been reported that the standard post-mortem examination has a low sensitivity $(47 \%)$ for detection of tuberculous lesions [27]. The less than perfect sensitivity of both the SICTT and abattoir surveillance will lead to the misclassification of infected animals as not infected, leading to an underestimate of the movement effect in this study. It is also important to note that this study investigated cattle moving from source to receiving herds in officially recorded moves. Officially recorded movements were those notified by herd owners to DAERA, with the vast majority being sales of cattle directly to other farmers or through markets. Unofficial movements which could include practices such as unrecorded borrowing or lending of bulls are not factored into the design of this study. Unrecorded movements would lead to an underestimation of the movement effect in this study as they would initiate infections which would have then been attributed to other sources. Green et al. used a model to estimate movement-based 
transmission of bTB in British cattle. In their work, they stated that $9 \%$ of herd transmissions were unexplained; this group included infections where source was spread from unrecorded movements [6].

\section{CONCLUSIONS}

Farms in Northern Ireland tend to be geographically fragmented and frequently have a business model dependent on trading of live animals with obvious epidemiological disease consequences [3]. Veterinary investigations into the source of confirmed bTB breakdowns in Northern Ireland (2002-2015) indicated that no source was established in $31 \%$ of cases, $29 \%$ were due to local spread, $15 \%$ were due to badgers, $14 \%$ were due to purchased animals, $7 \%$ were due to recrudescence and 4\% from other sources [18]. Although this study reported a lower percentage of breakdowns directly attributed to the purchase of bTB-infected animals $(6 \cdot 4 \%)$, they still equated to almost one in 15 bTB breakdowns, making this a significant source of infection. It is also important to acknowledge that the purchase of infected animals will not always lead to the same outcome in different herds, and at an individual herd level, there could be great variability in the breakdowns initiated from this source. For this reason, it is important that policy makers take cognisance of this source of infection and apply rational options to combat it. Employment of mitigating measures such as risk-based trading options is an especially useful tool as they engender an empowered culture where decisions can be implemented based on fact.

\section{SUPPLEMENTARY MATERIAL}

The supplementary material for this article can be found at https://doi.org/10.1017/S0950268817002424.

\section{ACKNOWLEDGEMENTS}

The authors would like to thank Roly Harwood for his constructive comments on the manuscript. This research received no specific grant from any funding agency, commercial or not-for-profit sectors.

\section{DECLARATION OF INTEREST}

None.

\section{REFERENCES}

1. Radostits OM, et al. Diseases associated with bacteria iv. In: Radostits OM, Blood DC, Gay CC, Hinchcliff KW, Constable PD, eds. Veterinary Medicine $A$ Textbook of the Diseases of Cattle, Horses, Sheep, Pigs and Goats. Philadelphia: Saunders-Elsevier, 2007, pp. 1007-1017.

2. DAERA, Statistical review of Northern Ireland agriculture 2015. (http://www.daera-ni.gov.uk/sites/default/ files/publications/dard/stats-review-2015-final-amended. PDF). Accessed 1 December 2016.

3. Abernethy DA, et al. The Northern Ireland programme for the control and eradication of Mycobacterium bovis. Veterinary Microbiology 2006; 112: 231-237.

4. DAERA, Tuberculosis disease statistics - December 2015. (http://www.daera-ni.gov.uk/publications/tuberculosisdisease-statistics-northern-ireland-2015). Accessed 1 December 2016.

5. Clegg TA, et al. Potential infection-control benefit for Ireland from pre-movement testing of cattle for tuberculosis. Preventive Veterinary Medicine 2008; 84: 94-111.

6. Green DM, et al. Estimates for local and movementbased transmission of bovine tuberculosis in British cattle. Proceedings of the Royal Society of London B: Biological Sciences 2008; 275: 1001-1005.

7. O'Hagan MJ, et al. Herd-level risk factors for bovine tuberculosis and adoption of related biosecurity measures in Northern Ireland: a case-control study. Veterinary Journal 2016; 213: 26-32.

8. Johnston WT, et al. Herd-level risk factors associated with tuberculosis breakdowns among cattle herds in England before the 2001 foot-and-mouth disease epidemic. Biology Letters 2005; 1: 53-56.

9. Reilly LA, Courtenay O. Husbandry practices, badger sett density and habitat composition as risk factors for transient and persistent bovine tuberculosis on UK cattle farms. Preventive Veterinary Medicine 2007; 80: 129-142.

10. Carrique-Mas JJ, Medley GF, Green LE. Risks for bovine tuberculosis in British cattle farms restocked after the foot and mouth disease epidemic of 2001. Preventive Veterinary Medicine 2008; 84: 85-93.

11. Wolfe DM, et al. The risk of a positive test for bovine tuberculosis in cattle purchased from herds with and without a recent history of bovine tuberculosis in Ireland. Preventive Veterinary Medicine 2009; 92: 99105.

12. Ramirez-Villaescusa AM, et al. Risk factors for herd breakdown with bovine tuberculosis in 148 cattle herds in the south west of England. Preventive Veterinary Medicine 2010; 95: 224-230.

13. Berrian AM, et al. Risk of bovine tuberculosis for cattle sold out from herds during 2005 in Ireland. Veterinary Record 2012; 170: 620.

14. Houston R. A computerised database system for bovine traceability. Scientific and Technical Review of the Office International des Epizooties (Paris) 2001; 20: 652-661.

15. Dohoo IR, Martin SW, Stryhn H. Logistic regression. In: Dohoo I, Martin SW, Stryhn H, eds. Veterinary 
Epidemiologic Research. Charlottetown, Prince Edward Island, Canada: AVC Inc, 2009, pp. 355-356.

16. Raftery E. Bayesian model selection in social research. Sociological Methodology 1995; 25: 111-163.

17. Dohoo IR, Martin SW, Stryhn H. Measures of association. In: Dohoo I, Martin SW, Stryhn H, eds. Veterinary Epidemiologic Research. Charlottetown, Prince Edward Island, Canada: AVC Inc, 2009: pp. 126-130.

18. DAERA. Bovine tuberculosis in Northern Ireland 2015 Report. (http://nics.intranet.nigov.net/daera/documents/tb-annual-report). Accessed 1 December 2016.

19. Menzies FD, Neill SD. Cattle-to-cattle transmission of bovine tuberculosis. Veterinary Journal 2000; 160: 92-106.

20. Menzies FD, et al. A matched cohort study investigating the risk of Mycobacterium bovis infection in the progeny of infected dams. The Veterinary Journal 2012; 194: 299-302.

21. McIlroy SG, Neill SD, McCracken RM. Pulmonary lesions and Mycobacterium bovis excretion from the respiratory tract of tuberculin reacting cattle. Veterinary Record 1986; 118: 718-721.
22. Donnelly CA, Nouvellet $\mathbf{P}$. The contribution of badgers to confirmed tuberculosis in cattle in high-incidence areas in England. PLoS (Public Library of Science) Current Outbreaks 2013; 5: 1-20.

23. Adkin A, et al. Assessing the impact of a cattle riskbased trading scheme on the movement of bovine tuberculosis infected animals in England and Wales. Preventive Veterinary Medicine 2016; 123: 23-31.

24. Adkin A, et al. Development of risk-based trading farm scoring system to assist with the control of bovine tuberculosis in cattle in England and Wales. Preventive Veterinary Medicine 2016; 123: 32-38.

25. DAERA. TB strategic partnership group's bovine TB eradication strategy for Northern Ireland information note. (http://nics.intranet.nigov.net/daera/documents/ tb-strategic-partnership-group $\% \mathrm{E} 2 \% 80 \% 99 \mathrm{~s}$-bovine-tberadication-strategy-ni-information-note). Accessed 1 January 2016.

26. Monaghan ML, et al. The tuberculin test. Veterinary Microbiology 1994; 40: 111-124.

27. Corner LA. Post mortem diagnosis of Mycobacterium bovis infection in cattle. Veterinary Microbiology 1994; 40: $53-63$. 